\title{
Le pouvoir des mots dans le nouveau consensus du développement
}

Un nouveau mode de gouvernement des pauvres

Jaime Marques Pereira et Pepita Ould-Ahmed

\section{(2) OpenEdition}

\section{Journals}

Édition électronique

URL : http://journals.openedition.org/ei/230

DOI : 10.4000/ei.230

ISSN : 2553-1891

Éditeur

Association Économie et Institutions

Édition imprimée

Date de publication : 30 mai 2010

Pagination : 5-14

ISSN : $1775-2329$

Référence électronique

Jaime Marques Pereira et Pepita Ould-Ahmed, «Le pouvoir des mots dans le nouveau consensus du développement », Économie et institutions [En ligne], 14 | 2010, mis en ligne le 31 janvier 2013, consulté le 03 mai 2019. URL : http://journals.openedition.org/ei/230 ; DOI : 10.4000/ei.230 


\section{Présentation du numéro}

Le pouvoir des mots dans le nouveau consensus du développement: un nouveau mode de gouvernement des pauvres

\section{Jaime Marques Pereira et Pepita Ould Ahmed}

Depuis le début des années 1980, les politiques, autant que les recherches consacrées au développement, ont connu une rupture radicale. D'une part, en effet, l'épuisement des politiques d'industrialisation et de croissance à caractère "autocentré ", déployées à partir de la fin des années 1940 dans nombre de pays " en voie de développement ", y a entraîné de nouvelles stratégies de transformation économique et sociale, où la promotion de la propriété privée et la "libéralisation " des prix, des échanges et des mouvements de capitaux tiennent désormais une place centrale. D'autre part, les réformes économiques mises en œuvre dans des pays à structures politiques aussi distinctes, voire opposées, que la Chine communiste (dès 1978), l'Inde (à partir de 1984), le Mexique et la Turquie (au milieu des années 1980), l'Iran islamique (depuis 1989), ou encore l'Egypte (depuis la fin des années 1980) sont quelques exemples saillants, parmi bien d'autres, qui portent témoignage de la convergence, bien avant l'effondrement du système soviétique (en 1991), des politiques de développement vers des modèles économiques et sociaux ayant pour matrice commune "l'économie de marché " (plus ou moins libéralisée selon les pays).

Cet infléchissement et convergence des politiques économiques et sociales entrent en interaction avec l'évolution des idées et des mots consacrés au développement. Cette interaction est devenue un champ d'analyse qui semble s'imposer pour caractériser cette convergence. Celle-ci interpelle le chercheur sur le sens sociologique d'un consensus intellectuel sur l'idée que ce dernier est une technologie du développement. L'éclipse des modèles de développement à orientation "radicale " (d'inspiration marxiste et/ou nationale...) a pour corollaire l'abandon de leurs présupposés doctrinaux, idéologiques et théoriques : d'où la disparition massive, dans la littérature économique hétérodoxe axée sur le développement, des catégories analytiques, à vocation à la fois positive (comme l'"échange inégal " et l'" accumulation dépendante ") et normative (comme le "changement social"), immanentes à ces modèles et dominantes jusqu'à la fin des années 1970. Aujourd'hui, on parle 
moins "d'échange inégal " et plus "d'ajustement structurel ", moins de "politique industrielle " et plus de "stabilité macroéconomique ". A l'orée des années 1990, des nouvelles politiques s'imposent à partir de nouveaux mots d'ordre comme ceux de "capital social ", "démocratie ", "gouvernance ", " développement durable ", "capabilities ", "participation ", "société civile ", "genre ", " solidarité ", ou encore "droits" (de l'homme, humains, sociaux, de propriété...), " empowerment ", " microcrédit "... Ces nouveaux mots sont l'expression de politiques de développement (" développement durable ", " microcrédit "...) et d'une certaine philosophie de l'action (" empowerment ", " capabilities ", " participation ", "société civile "). En effet, ce vocabulaire n'est évidemment pas neutre dans la mesure où il est l'indicateur des façons de définir (de poser) la question du développement - et par conséquent de concevoir les priorités et les politiques à mener dans les pays concernés. Le pouvoir des mots de mettre en forme les maux du développement, c'est-à-dire de les porter à l'existence d'une certaine manière et pas d'une autre, charrie nécessairement des enjeux décisifs. Il n'est ainsi pas étonnant dans ces conditions qu'ils soient l'objet de grandes rivalités entre les acteurs académiques, politiques et professionnels qui parlent au nom du développement.

Pourtant, phénomène paradoxal, il semble que les mêmes mots et les mêmes politiques s'imposent partout et font "consensus", si l'on en juge par l'application systématique des mêmes politiques économiques dans tous les pays du Sud quelque soit leur contexte économique, social, politique, historique, culturel, etc. et par l'usage du même vocable par l'ensemble des acteurs qui structurent le champ du développement (organisations financières internationales, gouvernements nationaux, ONG, chercheurs, experts, humanitaires, associations...).

L'objectif de ce numéro spécial est alors double : comprendre le pouvoir performatif des mots et préciser la nature de ce consensus dans l'action collective. D'une part, il s'agit d'interroger les mots eux-mêmes, leurs significations, leurs usages, leur arrièreplan idéologique et leur pouvoir performatif, dans le cadre des espaces politique, économique et social dans lesquels ils se déploient. Les mêmes vocables sont utilisés dans différentes sphères - disciplinaires, professionnelles, politiques, linguistiques, culturelles, juridiques, etc. - mais n'ont pas forcément le même sens : il peut y avoir désaccord de divers points de vue : idéologique, étymologique, fonctionnel... Dans le domaine du développement, ces sphères sont en interaction, et il peut en découler des malentendus, des désaccords, voire des conflits de reconnaissance, de prééminence, non seulement autour du sens d'un mot, mais aussi entre les corps disciplinaire, institutionnel et professionnel qui le 
promeuvent. Dans cette perspective, qui est le plus légitime à dire le sens de chaque terme? Qui a la puissance de s'approprier, normaliser une définition, l'imposer aux acteurs d'un domaine? Car, à peine "produit ", chaque mot constitue, potentiellement, un objet de discorde et, partant, un opérateur de conflits de légitimité et d'hégémonie.

D'autre part, il s'agit d'interroger la convergence qui semble se nouer autour des vocables et des politiques du développement. Pour ce faire, ce numéro spécial, consacré aux mots du développement, se propose d'analyser les rapports qui lient les différents acteurs du développement entre eux afin d'évaluer la nature du "consensus " et les conditions d'émergence de ce "consensus". Une attention particulière est portée, d'une part, au choix des acteurs du développement reconnus comme "légitimes" par le champ du développement et à leur degré de légitimité respective pour peser sur les mots et les politiques du développement; d'autre part, au dispositif ou à la mise en scène institutionnelle, politique et médiatique permettant que la parole et les désaccords sur le développement s'expriment. Afin de mieux apprécier la part d'adhésion, de consentement et/ou de contrainte qui lie les acteurs entre eux au nom du développement, il faut retracer les trajectoires et les usages des vocables et mettre au jour les médiations au travers desquelles une fraction de ces agents forge et façonne les notions du développement, tandis que d'autres les reçoivent, les intègrent, ou, au contraire, les réinterprètent.

Cette interrogation sur les mots eux-mêmes et le rapport des acteurs à ces mots a fait l'objet d'un colloque pluridisciplinaire ${ }^{1}$. Les paragraphes qui précèdent rappellent brièvement sa problématique. Le dossier, ici présenté, rassemble des contributions de sociologues et d'économistes, prenant en compte à la fois la configuration discursive actuelle du développement et leurs politiques économiques mais aussi l'analyse concrète des pratiques des différents acteurs impliqués dans des "actions " de développement ou dans la recherche "sur" le développement. Il comprend cinq communications dont l'ensemble contribue, nous semble-t-il, à mettre en évidence la réalité, observable dans le jeu des acteurs, d'une nouvelle forme d'action institutionnelle réputée promouvoir le développement par les incitations à l'intégration des pauvres à l'économie de marché. Les enseignements pour le débat économique

1 "Les mots du développement: genèse, usages et trajectoires " qui s'est tenu les 13 et 14 novembre 2008 à l'université Paris-Dauphine, http://calenda.revues.org/nouvelle11306.html. D'autres communications ont été publiées dans un numéro spécial de la revue Tiers-Monde : "Les mots du développement. Trajectoires et pouvoirs ", $n^{\circ}$ 200, octobre-décembre 2009 
autour des institutions ne constituent pas la trame centrale du dossier mais sont perceptibles à la croisée des articles.

\section{Derrière de nouveaux mots, une même politique et philosophie de l'action}

Fortement critiquées par les gouvernements du sud et l'opinion internationale après les crises post-ajustement structurel, les institutions et bailleurs de fonds internationaux, grands producteurs de mots et de discours, mais également instigateurs dans la pratique des politiques de développement, ont opéré à partir de la fin des années 1990 un changement de lexique pour parler du développement. Les termes comme " démocratie ", "société civile " (à savoir les citoyens, les communautés, les associations, les coopératives, mais aussi les ONG), "participation " (correspondant à une injonction à faire quelque chose), "empowerment ", " développement local ", " microcrédit ", " capabilities ", "les politiques par le bas" ("to create economic and social development from below ")... sont devenus les nouveaux termes emblématiques de leurs discours officiels.

L'emploi de nouveaux mots accompagne un changement de paradigme économique. A partir de l'an 2000, le rôle des institutions occupe désormais une place importante dans les problématiques théoriques du développement. Cette prise en compte des institutions s'effectue par ailleurs dans un contexte général de reconnaissance de leur rôle en économie grâce au succès que connaît le néoinstitutionnalisme. La littérature économique dominante sur le développement multiplie ses efforts pour identifier les "bonnes" institutions (définies sous le seul critère de l'efficience) qui permettraient de sortir de la pauvreté. La Banque mondiale s'inscrit également dans ce mouvement et opère à son tour sa conversion aux institutions. Le texte de Benoît Prévost revient particulièrement sur l'évolution des discours et de la doctrine de cette organisation. Celleci appréhende la question du développement à partir de l'articulation de l'équité, l'égalité des chances et l'empowerment, sous l'influence de trois mouvements théoriques: l'influence de la philosophie moderne en économie, en particulier avec les travaux d'Amartya Sen et ceux de John Rawls ; la réactivation de travaux sur les inégalités, la pauvreté et le développement (sous l'influence de François Bourguignon à la Banque mondiale); enfin, la prise en compte des institutions pour mettre en place une "bonne gouvernance ". Le texte de Benoît Prévost montre que les documents de travail, séminaires, colloques, mais aussi les discours officiels de la Banque mondiale comme ceux d'ailleurs des autres grandes organisations financières et bailleurs de fonds internationaux - affichent une revendication 
d'un certain égalitarisme libéral dans la manière de concevoir le développement et des politiques à mener, à travers les notions "d'autonomie " et de "responsabilité " des personnes pauvres.

Cependant il ne faut pas s'y tromper. Si le vocable employé par les organisations internationales connaît un changement incontestable, le changement des politiques de développement et de leurs arrière-plans idéologiques, quant à lui, est beaucoup moins évident ${ }^{2}$. En effet, la continuité des politiques d'ajustement structurel est en réalité toujours affirmée par les organisations financières internationales (Banque Mondiale, FMI), mais sur la base d'une nouvelle rhétorique et de nouvelles modalités d'intervention. La prise en compte des problématiques institutionnelles se traduit en particulier par une reformulation de la conditionnalité: les nouvelles propositions d'intervention consistent désormais à ne plus intervenir directement auprès des autorités nationales pour leur imposer un programme d'ajustement structurel, mais à les encourager à concevoir leurs propres programmes. Sans interférer ainsi, explicitement, dans le choix des politiques publiques, elles se limiteraient, implicitement, à conditionner leurs aides en fonction des résultats et des objectifs économiques affichés par les autorités nationales et de la mise en place des "bonnes " institutions par les pays aidés. On passe ainsi, à la fin des années 1990, d'une conditionnalité exprimée essentiellement en termes de politiques macroéconomiques à une conditionnalité exprimée en termes de construction institutionnelle (Lafaye de Micheaux, Ould-Ahmed, 20073).

Si l'évolution des mots n'indique pas véritablement un changement idéologique des politiques de développement, elle révèle en revanche les fondements philosophiques de l'action individuelle et des politiques que les organisations internationales soutiennent. De ce point de vue, le concept d'empowerment, devenu l'un des mots clés du nouveau discours sur le développement, est éclairant. Entendu comme l'" expansion de la liberté de choix et d'action " (Banque mondiale, 2002, cité dans Prévost, p.19) ou comme

2 Se reporter à l'ouvrage collectif Institutions et Développement. La fabrique institutionnelle et politique des trajectoires de développement, co-dirigé par $\mathrm{E}$. Lafaye de Micheaux, E. Mulot et P. Ould-Ahmed en 2007 aux PUR, qui, à partir d'une lecture institutionnaliste d'économie politique du développement, analyse l'évolution des politiques de développement et le poids des différents acteurs économiques ainsi que celui des Etats souverains à mettre en œuvre ces politiques.

3 Lafaye de Micheaux E., Ould-Ahmed P., 2007, "Les contours d'un projet institutionnaliste en économie du développement " in E. Lafaye de Micheaux, E. Mulot et P. Ould-Ahmed (eds), Institutions et développement. La fabrique institutionnelle et politique des trajectoires de développement, PUR, p. 9-37. 
" renforcement de la capacité d'une personne à faire des choix (...) et à les transformer en actions et résultats désirés ", l'empowerment reflète le soutien accordé par les organisations financières internationales aux politiques de développement ou mesures "portées par le bas" telles que le microcrédit ou encore la microentreprise (cf. Fouillet et Pairault dans ce numéro). "Les pauvres sont ainsi appelés à devenir les acteurs de leur sortie de pauvreté, ce qui favorise leur autonomie, tout en étant in fine responsables de leur succès et de leur échec à partir du moment où les conditions auront été réunies pour assurer l'égalité des chances " (Prévost, p. 9). Aussi, à travers le soutien de réformes institutionnelles, économiques et financières visant à renforcer la capacité des pauvres à disposer davantage d' "autonomie " et de " responsabilité ", c'est la philosophie libérale de l'action individuelle qui se trouve ainsi réaffirmée. Il reste à voir comment ces termes font consensus et quelles en sont les modalités.

\section{Production et imposition des mots : Comment s'imposent- ils? Consensus?}

Ce dossier donne à voir et analyse des mots qui s’imposent et font croire à un consensus par leur usage commun tant par les financiers, les concepteurs que par les praticiens. Il en découle une prise de parole contrôlée des personnes concernées par les projets de développement.

Se pose donc la question de savoir comment les nouveaux acteurs de l'univers professionnel du développement sont porteurs d'un consensus intellectuel sur les moyens du développement et ce qu'il est, consensus réputé refléter un consensus social produit par les techniques de participation. Le dossier montre qu'il faut s'interroger sur l'existence même de ce consensus intellectuel tant en termes théoriques que pratiques, et plus encore de sa possibilité de refléter ou de générer un véritable consensus social: existe-il vraiment ? Si oui, quelle est sa nature ? N'est-il que contrôle de la parole publique ? Ces questions sont d'abord posées au regard de l'analyse de l'appropriation de ces mots et du détournement de leur sens. Les communications ici rassemblées suggèrent une véritable économie politique et symbolique des entrepreneurs du développement, montrant en particulier comment le pouvoir des mots dans leur circulation économique et politique opère la persuasion. Il faut donc certes se poser la question de l'existence d'un certain consensus pragmatique entre les professionnels du développement et voire même entre les usagers des projets. De ce point de vue, le texte d'Amin Allal est particulièrement illustratif de cette question, étudiant le lien entre les intérêts économiques et les intérêts symboliques (valorisation de soi). 
D'un point de vue théorique, la question du consensus est encore mal cernée puisqu'on observe d'un côté une force de consensus et de l'autre l'expression de désaccords. On voit bien là comme le rappelle Bruno Lautier que consensus n'est pas consentement. La différence déplace le problème sur ce que l'on peut appeler avec Benoît Prévost un art de la persuasion dans la production d'un consensus intellectuel qu'a signifié le consensus de Washington. On comprend que cet art visait à étouffer la portée de ces critiques et à masquer ainsi la continuité de l'ajustement structurel en renouvelant la théorie du rapport d'équité-justiceégalité des chances grâce à de "bonnes institutions " qui pallient les défauts du marché par leur effet d'empowerment. Cela dit, c'est un programme d'occultation mais l'idée n'est qu'une déduction des contradictions logiques repérables dans l'exposé d'une technique de persuasion. Cette idée n'est qu'une hypothèse qui mérite alors d'être davantage élaborée d'un point de théorique pour en préciser la portée analytique, et pouvoir alors la vérifier dans des cas empiriques divers.

La généalogie que propose Bruno Lautier de la pensée du consensus resitue son sens actuel de fabrique d'une représentation collective. Cette fabrication est analysée dans divers jeux d'acteurs qui transcendent les réalités nationales car ils semblent constituer des aspects structurels d'une gestion politique mondialisée des populations pauvres (fort bien illustrés par les différentes contributions de ce numéro spécial). Un premier jeu présenté ici par Blandine Destremau est celui des réunions d'experts du BIT visant à définir les outils d'enquête appropriés à l'appui au travail des femmes pauvres dans le monde arabe. Le second travail, d'Amin Allal, porte sur le montage au Maroc et en Tunisie de ce que l'auteur appelle un tamis participatif. L'article de Cyril Fouillet et Thierry Pairault, quant à lui, compare la forte croissance décentralisée de la microfinance en Inde et l'organisation étatique du microcrédit en Chine. L'intérêt de la comparaison est de mettre en lumière la convergence de l'instrumentalisation politique. Le moins libéral des deux pays en la matière se voit offrir la vice-présidence de la Banque mondiale. Les mots semblent avoir des vertus semblables comme opérateurs d'une sélection des acteurs qui, de façon récurrente dans chaque situation, est le vecteur d'une dépolitisation. Celle-ci apparaît à l'échelle locale comme une cooptation antipolitique (c'est-à-dire qui contourne les réseaux électoraux) ou encore elle concerne la "gouvernance " mondiale au travers de l'intégration de la Chine au centre concepteur de cette fabrique du consensus.

Le texte de Bruno Lautier s'attache particulièrement à préciser les malentendus du consensus et insiste sur sa fonction politique de clôturer le débat, voir d'empêcher la prise de parole elle- 
même. "Les consensus ne servent pas tant à réaffirmer l'accord de leurs parties prenantes qu'à clore toute délibération: ni ceux qui sont dans le consensus, ni ceux qui le contestent, n'auront droit à une parole légitime" (Lautier, p. 65). L'auteur montre la nature profondément politique du consensus et pour ce faire, il réalise une généalogie sélective du terme consensus (à partir de Spinoza, Comte, Durkheim, Tocqueville...), une histoire de ce mot, de ses significations dans la langue française, mais aussi de ses usages par les différentes disciplines (philosophie morale, sociologie, psychologie sociale, économie) et dans le développement (Consensus Building Institute). Le consensus semble produit par des délibérations ou encore des processus de normalisation qui conduisent à des positions médianes. Il peut être aussi l'expression d'un certain nombre d'opinions convergentes. Il apparaît ainsi comme une procédure garantissant le pouvoir d'une minorité d'imposer à la majorité les sujets dont il faut discuter. Ainsi le consensus est érigé en technique du consensus building qui est employé d'abord par le BIT et qui s'est ensuite propagé aux autres organismes internationaux qui le promeuvent comme condition de la stabilisation. En s'abstenant de nommer clairement l'objet même du consensus, on consacre ainsi l'asymétrie Nord-Sud dans l'énonciation de la conditionnalité.

La technique normalisée du consensus building pour reprendre le terme de la littérature internationale que Bruno Lautier passe en revue est examinée par Blandine Destremau à l'échelle d'une organisation internationale, le BIT. L'étude de cas présentée par cette auteure montre comment s'organise la construction collective d'un savoir consensuel entre exécutants des politiques publiques de genre au Moyen Orient. Les mots sont ici négociés, de leur traduction statistique à la communication dans l'enquête. Son texte rapporte une expérience de collaboration avec un bureau régional du BIT au cours de laquelle un certain nombre de normes concernant le travail et la protection sociale devait être incorporé dans un programme appliqué et conçu au niveau régional des Etats arabes et dans les pratiques d'acteurs institutionnels. L'auteure pose la question de l'efficacité et de l'ambivalence des techniques de diffusion des normes construites autour des procédures participatives. Elle montre que les négociations entre participants ont pour nécessité affichée (obligation) de déboucher sur une adhésion consensuelle au nom d'un bien commun, et ce malgré des désaccords et différends entre les participants jugés finalement secondaires, et non sur un débat substantiel à prendre au sérieux : "Finalement nous étions tous là pour le bien de tous, du même côté, l'ennemi commun ayant été désigné : la précarité, la pauvreté, l'insécurité " (Destremau, p. 91). L'objectif de ce programme est de diffuser des normes universelles, en particulier celle de "travail 
décent " et d' "emplois productifs " pour réduire la pauvreté et les inégalités de genre et contribuer à parvenir à un développement durable dans les pays arabes. Son texte montre comment une norme est imposée sans donner l'air de l'être (grâce à la mise en scène d'un dispositif de participation). En effet, l'analyse du dispositif de participation et de collaboration entre les différentes catégories d'experts met au jour "le rapport de force larvé qui s'est instauré, dans le cadre de la mise en œuvre de ce programme, entre une organisation internationale dont la mission est largement normative, et un certain nombre d'acteurs institutionnels, supposés changer leurs pratiques et référents pour les rendre plus conformes aux normes universelles et consensuelles portées par l'organisation, tout en étant invités à participer à l'élaboration de leur contenu " (Destremau, p. 74).

\section{Circulation et réception des mots: Comment sont-ils véhiculés, perçus, réceptionnés, instrumentalisés?}

Le texte d'Amin Allal revient sur le terme de "participation" qui renvoie à une idée et à un dispositif de réformes supposées produire à la fois le développement et la "démocratisation " des pays du Sud. La référence à la "participation" apparaît de façon importante dans les rapports de la Banque Mondiale et du PNUD au début des années 1990, et se décline par la suite dans les rapports nationaux des développeurs. La participation au développement par la " société civile " se traduit concrètement par la mise en place de commissions dites "participatives" (nationales, régionales ou locales), par la création d'associations... Amin Allal s'intéresse particulièrement à ces acteurs qui composent ces associations ou collectifs dits participatifs, en prenant comme terrain d'observation le Maroc (à Rabat) et la Tunisie (à Tunis). Si pour les organisations internationales et les bailleurs de fonds, le discours de la " participation ", comme celui par ailleurs de "l'empowerment ", est un discours d'injonction à faire quelque chose, l'auteur montre la diversité des intérêts et des représentations que les acteurs locaux $\mathrm{du}$ développement international ont de leurs propres pratiques (représentants des bailleurs de fonds, des ONG internationales et des associations, élus locaux et cadres des ministères concernés par les projets de développement). Au terme de son analyse il montre comment les processus de sélection des acteurs dans le cadre des dispositifs dits participatifs conduisent à une cooptation antipolitique des acteurs de la "participation ", même si ces derniers préfèrent appréhender leur participation comme l'expression d'une "démocratisation " de la société. 
Le texte de Cyril Fouillet et Thierry Pairault montre comment le terme mais aussi la pratique de la microfinance sont réceptionnés et réappropriés par les Etats et les organisations locales qu'ils étudient (Chine et Inde). En décrivant la normalisation politique des pratiques financières de la microfinance, leur texte donne à voir une diversité des modalités d'appropriation de cette pratique par les Etats et les acteurs locaux, intégrant des considérations politiques, sociales, voire religieuses. Les autorités chinoises affichent une certaine méfiance à l'égard de la croyance de l'omnipotence de l'intérêt privé pour satisfaire des besoins collectifs. Elles préfèrent organiser les institutions de la microfinance dans le cadre des communautés urbaines et rurales dont elles promeuvent l'organisation. Compte tenu de la portée politique de la nomination des pratiques, le terme microfinance ne trouve aucune traduction littérale en chinois. Celui le plus souvent adopté pour désigner les activités de la microfinance signifie "prêt chirographaire de faible montant " (p. 129), soit microcrédit au sens strict, excluant ainsi toute forme de microfinance. Le gouvernement chinois rejette toute forme relativement autonome de microfinance afin de garder le contrôle macroéconomique et social. L'impressionnante expansion récente de la microfinance en Inde paraît plus proche du modèle libéral par sa décentralisation mais on comprend qu'il s'agit juste d'une décentralisation de son appropriation, ce qui n'est guère étonnant dans un système fédéral. Ici aussi, la structure traditionnelle du pouvoir politique semble confortée, tant "sa dimension populiste et démagogique est évidente" (p.142). L'instrumentalisation politique qu'informe cette comparaison paraît exemplaire du chemin institutionnel d'une production d'un consensus qui n'en est pas un - du cœur cognitif opérationnel de la technique de persuasion à ceux qui réorganisent des "capabilities " sur tout type de terrain.

La dernière phrase de l'article de Pairault et Fouillet pointe l'enjeu proprement économique des idiosyncrasies nationales, régionales et locales s'appropriant des best practices. "L'écueil actuel pourrait être non plus celui du consensus de Washington, mais celui du consensus qu'imposeraient les États à leurs circonscriptions territoriales ou encore l'absence de consensus national faute aux États de pouvoir en instaurer un" (p.143). L'usage de mots et les pratiques d'un des domaines emblématiques de la gouvernance que constitue la microfinance ne semble pas avoir l'effet attendu. L'objectif d'un accroissement de la pratique de la liberté comme capacité sociale à créer une offre marchande semble irréel quand les mots et les pratiques sont le moyen de sauvegarder des modes établis de contrôle politique par une nouvelle forme de gouvernement des populations pauvres. 